\title{
Shim-on-Chip Design for Microfluidic NMR Detectors
}

\author{
S. G. J. van Meerten, P. J. M. van Bentum, and A. P. M. Kentgens*10 \\ Solid State NMR, Radboud University, Heyendaalseweg 135, Nijmegen, The Netherlands 6525 AJ
}

\section{Supporting Information}

ABSTRACT: In this contribution we present a novel system for shimming capillary samples such as used in microfluidic NMR probe heads. Due to the small sample size, shimming microliter samples using regular shim coils is complicated. Here we demonstrate the use of a series of parallel wires placed perpendicular to $B_{0}$ as a Shim-on-Chip shim system. This is achieved by placing a ribbon flat cable horizontally over the NMR detector, in our case a stripline. The current through each wire of the ribbon cable can be controlled
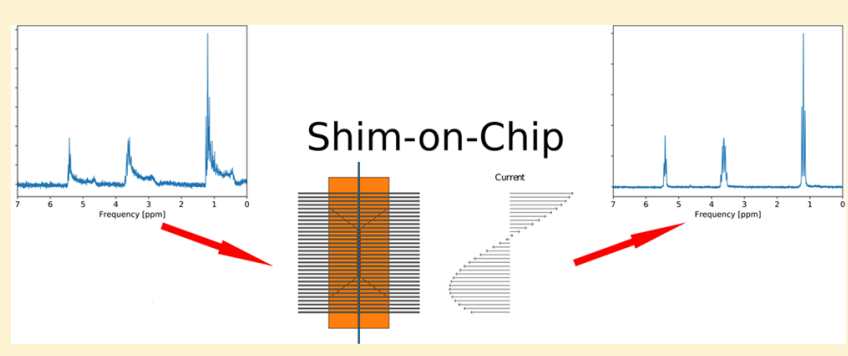
independently employing a 16 channel DAC. This makes for a simple, cheap, and easy to construct alternative to regular shim systems. The Shim-on-Chip is, nevertheless, quite flexible in creating a magnetic field which matches the inhomogeneity of the magnet in one dimension. The capillary sample geometry is well suited for this type of shimming since its length is much larger than its width. With this Shim-on-Chip system we have reached line widths of $2.2 \mathrm{~Hz}$ (at $50 \%$ ) and $27 \mathrm{~Hz}$ (at $0.55 \%$ ) on a $144 \mathrm{MHz}$ NMR spectrometer without any other room temperature shims. Unlike regular shims, the Shim-on-Chip is located inside the NMR probe. It is always centered on the NMR sample, because of this the shims have an intuitive effect on the line shape. Therefore, the manual shimming is simpler when compared to a regular shim system, as it is difficult to position a microliter sample in the exact center of the shim coils. We furthermore demonstrate the use of a Shim-on-Chip method in a $400 \mathrm{MHz}$ Rapid-Melt DNP system. Decent line widths were achieved even for a sample which is located off-center inside the NMR magnet.

Thhomogeneity in the magnetic field of an NMR magnet 1 broadens lineshapes and decreases their amplitude. This reduces both the resolution and the signal-to-noise ratio (SNR) of the NMR spectrum. The success of NMR is largely determined by both the homogeneity and the stability of the magnetic field. To obtain the required resolution in the NMR spectrum, the magnetic field has to homogenized. This process of homogenizing a magnetic field is called shimming. Shimming of an NMR magnet is performed by applying currents to various shim coils. ${ }^{1}$ These shim coils are generally located in the space between the NMR probe and the magnet. Each shim coil generates a magnetic field which resembles a specific spherical harmonic function. ${ }^{2-4}$ Any field profile can be decomposed into an infinite series of spherical harmonics, the multipole expansion. So the shim coils can be used to correct the field up to certain order depending on the number of shim coils present. For regular NMR samples (NMR tubes), this is a convenient method to homogenize the magnetic field over the NMR sample. However, for small samples used in microcoil detectors, these type of shim coils are located too far away from the sample. The small structures used in microcoils for microfluidic $\mathrm{NMR}^{5}$ generally cause relatively large gradients in the magnetic field, which means high electrical power is required for proper shimming. Also it is more difficult to position a microcoil sample in the exact center of the shim coils. This complicates the shimming process due to "mixing" of the shim profiles. ${ }^{6}$ Because of these effects, the regular NMR shim coils are less suitable for shimming capillary samples, particularly for microfluidic probes with microcoil detectors as is discussed here.

In our lab a stripline microcoil NMR detector has been developed. ${ }^{7}$ In this setup, the sample is contained in a fused silica capillary with an outer diameter of $360 \mu \mathrm{m}$ and an inner diameter between 100 and $250 \mu \mathrm{m}$. The active region of the employed stripline detector has a length of $8 \mathrm{~mm}$. Since the length of sample is much larger than the width the shimming of a capillary sample is a nearly 1-dimensional problem. In this manuscript, we demonstrate the use of a Shim-on-Chip for shimming capillary samples.

When a current is passed through a wire it creates a magnetic field which decreases with the inverse of the distance $r$ from the wire. When multiples of these wires are placed parallel, an almost arbitrary field profile can be created in one dimension by controlling the current through each wire independently. These parallel wires can be placed close to the detector, as shown in Figure 1. This reduces the amount of current required for the shimming of microliter samples. Since a single Shim-on-Chip can generate a large number of different field profiles, only one simple "coil" geometry is required. This simplifies construction and reduces the amount of space required for the shim coils.

Received: $\quad$ May 22, 2018

Accepted: August 6, 2018

Published: August 6, 2018 

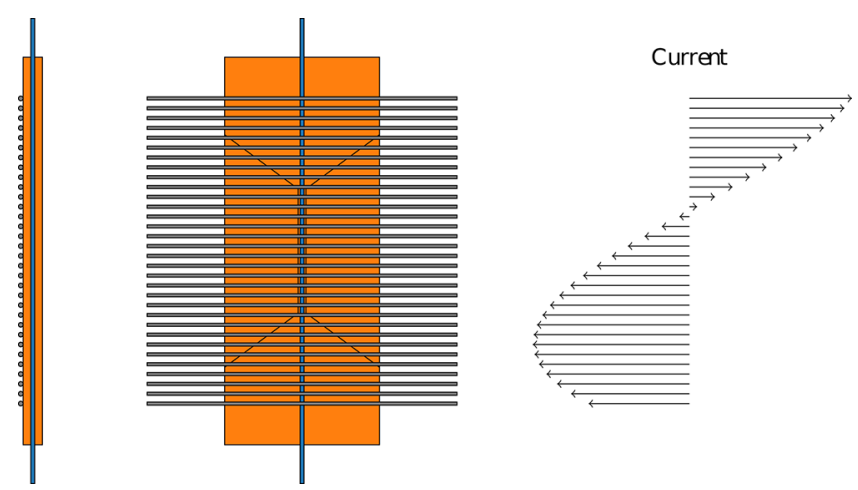

Figure 1. Schematics of the Shim-on-Chip setup. (Left) Side view of the stripline, capillary, ground plate, and shim-wires. (Middle) Front view without the ground plate. The current through each wire can be controlled independently.

\section{EXPERIMENTAL SECTION}

A stripline NMR probe consists of a stripline chip which is covered by a ground plate as shown in Figure 1. For the shim setup, a flat ribbon cable is placed on top of the ground plate, at the side where the sample is located. We employed a flat ribbon cable (BKL electronic) consisting of 40 wires of no. 30 AWG with a spacing of $0.635 \mathrm{~mm}$. An electrical diagram of the setup is given in the Supporting Information (Figure S1). On one end, all the wires of the ribbon cable are soldered together. On the other end the wires are connected through $220 \Omega$ resistances to a 16 channel, 16-bit DAC (Measurement Computing USB-3114) with a bipolar range of $\pm 10 \mathrm{~V}$. The wires are connected together in pairs where the current from one resistor is divided over two adjacent wires of the ribbon cable. The four outer wires on either side of the ribbon are connected to ground as a return path for the current. The ground wires are far away from the sensitive region, so their effect on the shimming is relatively small. Pictures of the Shim-on-Chip and the DAC are given in Figure S2 of the Supporting Information.

\section{SHIM PROFILES}

The current through the wires can be optimized sequentially, but for manual shimming this is not ideal. In optimizing the spectral line shape, there is a strong dependence between the parameters (the current through the individual wires). This complicates the "error landscape" and makes it difficult to find the global optimum. To simplify the optimization, the separate line currents can be combined into a more orthogonal basis set. A suitable basis set would be the polynomial functions $\left(z^{0}, z^{1}, z^{2}\right.$, etc.). This is because these functions change the line shape in a more intuitive way (similar to the spherical harmonics). Even though the polynomial functions are not completely orthogonal functions.

In Figure 2, the $z$-component of the magnetic field created by the Shim-on-Chip is shown in the $x z$-plane. The field of the NMR magnet is oriented along the $z$-direction as well. The current through the wire pairs are distributed linearly between $-40 \mathrm{~mA}$ and $40 \mathrm{~mA}$ from the bottom to the top, respectively. The magnetic field profile was calculated using the Biot-Savart law. For an infinitely long wire in the magnetostatic case, this gives a total magnet field of $B_{\text {shim }}=\frac{\mu_{0} I}{2 \pi r}$, where $B_{\text {shim }}$ is the magnetic field at position $r$ from the wire, $\mu_{0}$ is the permeability of vacuum, and $I$ is the current running through the wire. Using this equation the magnetic field $\left(B_{\text {shim }}\right)$ inside the capillary can
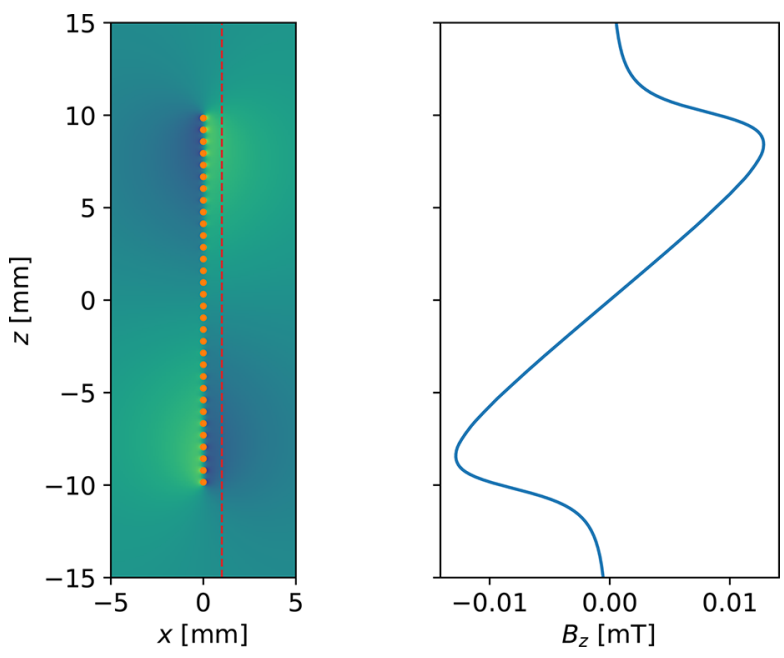

Figure 2. (Left) Calculated strength of $B_{z}$ in the plane perpendicular to the parallel wires. The current profile is linear over the wire pairs ranging from $40 \mathrm{~mA}$ through the top wire pair and $-40 \mathrm{~mA}$ through the bottom wire pair. The red dashed line shows the location of the capillary. (Right) Calculated field strength $\left(B_{z}\right)$ in the capillary.

be calculated. The field of the main magnet $\left(B_{0}\right)$ is orders of magnitude larger than the magnetic field generated by the shims. This means the vector components of $B_{\text {shim }}$ perpendicular to $B_{0}$ have a negligible contribution to the total field and can be dropped. The resulting $B_{z}$ generated by the wires as a function of position is shown in Figure 2 (right) for a linear current profile.

As can be seen from this curve, the magnetic field created does not follow the desired polynomial function (linear in $z$ ). For higher order polynomials, this deviation is even worse. It is more convenient to use a basis set where not the current but the actual magnetic field profile is described by the polynomial function $z^{n}$. To create these basis functions, an optimization procedure was performed. The current through each wire pair was used as a parameter. The squared difference between the simulated field and the desired $z^{n}$ profile was minimized in the active (sensitive) region $(8 \mathrm{~mm})$ of the detector. The currents were constrained to be symmetric or antisymmetric over the ribbon for even or odd values of $n$, respectively. With this constraint, the currents through the second half of the wire pairs can be determined from the first half, due to the even/odd symmetry. This leaves eight free parameters for the minimization (seven if normalization is included).

The relative currents to obtain the desired shim profiles are given in the Supporting Information for wire pairs 0 to 7. To obtain the values for the pairs 8 to 15 , the list should be reversed and the values multiplied by $(-1)^{n}$. In Figure 3, the optimized $z^{1}$ shim profile is shown. The shim profiles of the first six polynomials are given in Figure S3 with the actual currents tabulated in Table S1 of the Supporting Information. These optimized basis sets reduce the number of iterations needed to find the optimal shim settings, because of the reduced overlap of the profiles.

\section{AUTOMATED SHIMMING}

The Shim-on-Chip can be used in combination with automated shimming methods. The shim parameters can be optimized using a Nelder-Mead algorithm (simplex). ${ }^{8,9}$ In each iteration, the shims are set according to the algorithm. Then an FID is recorded and the negative of the FID surface is used as the costvalue of the minimization. The Nelder-Mead is a local 

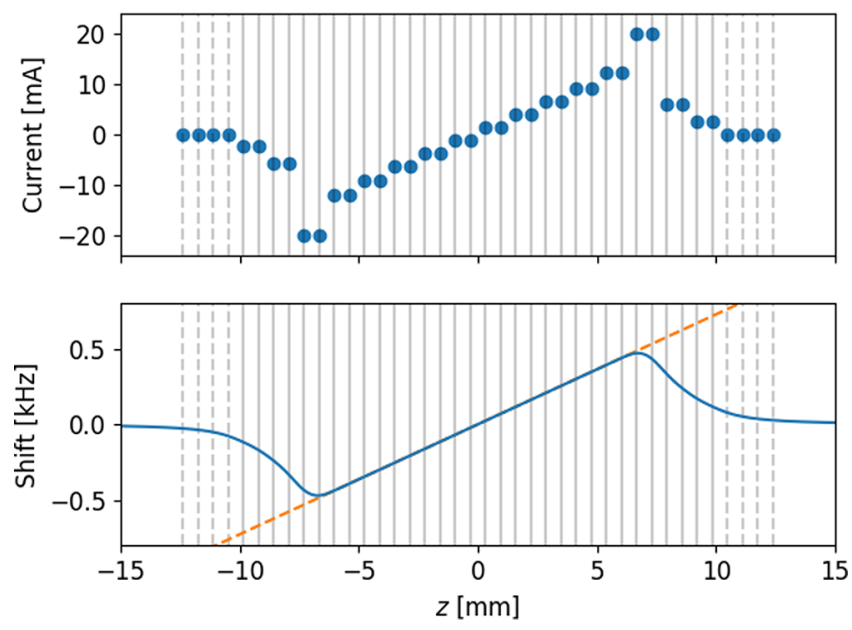

Figure 3. Field profile created by the optimized $z^{1}$ current profile. The top plot shows the currents through the wires. The bottom plot shows the position dependent shift in resonance frequency caused by these currents.

optimization algorithm, which means the choice of a basis set is important to prevent algorithm from getting stuck in a local minimum. For this reason, the currents cannot be used directly as parameters for such a minimization procedure. It is possible to use a global optimizer such as a differential evolution algorithm to prevent the local minimum problem, but the number of iterations required is much larger. ${ }^{10}$ This type of global optimization takes a couple of hours to complete, whereas the Nelder-Mead algorithm only takes $30 \mathrm{~min}$. Thus, the global optimizer is only suitable for the initial shimming, whereas a local optimization can be used to adjust the shims on a more regular basis.

In principle, it should also be possible to combine the Shimon-Chip with pulsed field gradient shimming. ${ }^{11}$ In pulsed field gradient shimming, an "image" is made of the magnetic field profile. ${ }^{12}$ When the magnetic field profile is known, it is easy to determine the currents required for the Shim-on-Chip to cancel out the inhomogeneity. Since the Shim-on-Chip is a onedimensional shimming method, only a pulsed $z$-gradient is required for pulsed field gradient shimming.

\section{RESULTS}

To test the Shim-on-Chip, experiments were performed on a $144 \mathrm{MHz}$ extra wide bore Infinity plus NMR spectrometer. The magnet is fitted with an 18 channel Oxford shim tube. So the performance of the Shim-on-Chip can be compared with that of regular shims. A sample of water was put in a capillary with an inner diameter of $100 \mu \mathrm{m}$. First the magnet was manually shimmed using the regular shims. Multiple spectra were recorded to also determine the stability of the shims. The resulting 20 spectra are shown in Figure 4a. The signal is modulated by the $50 \mathrm{~Hz}$ from the main power line passing through the shim power supply. This results in sidebands around the resonance at $\pm 50 \mathrm{~Hz}(\approx 0.35 \mathrm{ppm})$. The full width half maximum (fwhm) after shimming is approximately $2.4 \mathrm{~Hz}$. It should be noted that for shimming this sample the $\mathrm{Z} 2$ shim had to be turned to its maximum value. This is caused by the relative short length of the sample, which requires large gradients and thus large currents through the coils located far away from the sample.

In a follow-up experiment, the regular shims were turned off and the sample was shimmed using the Shim-on-Chip. The resulting spectra are shown in Figure $4 \mathrm{~b}$. With the Shim-onChip, a similar line width can be obtained as with the regular shims (fwhm of $2.2 \mathrm{~Hz}$ ). The Shim-on-Chip gives no visible 50 $\mathrm{Hz}$ modulation. This demonstrates the performance of the DAC in isolating the channels from the main power line.

Experiments were performed with and without the ribbon flat cable present. This showed that the presence of the ribbon cable itself gives no detectable disturbance in the local magnetic field in the capillary.

To demonstrate the use of the automated shimming a spectrum of pure ethanol was acquired in a capillary with an inner diameter of $100 \mu \mathrm{m}$. In Figure 5a, the spectrum of ethanol is shown without any shimming. The Shim-on-Chip was optimized using the global optimizer directly on the ethanol sample. After optimization of the Shim-on-Chip, the spectrum of Figure $5 \mathrm{~b}$ was obtained. The fwhm is $2.5 \mathrm{~Hz}$, which is enough to resolve all of the multiplet structure of the peaks. This shows that the automated optimization of the shims works well even on spectra with multiple resonances.

The stripline NMR detector is well suited for performing experiments using in-line or stopped-flow systems, since the sample is contained inside a capillary. In the next experiment, the NMR probe was connected to a stopped-flow system. First the

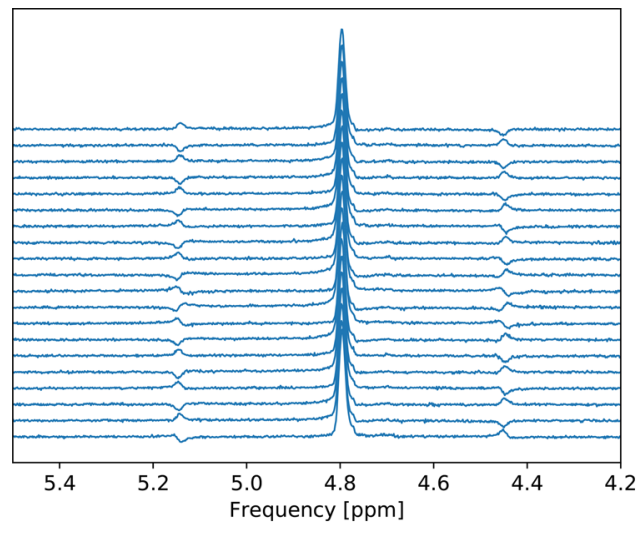

(a) Regular shims

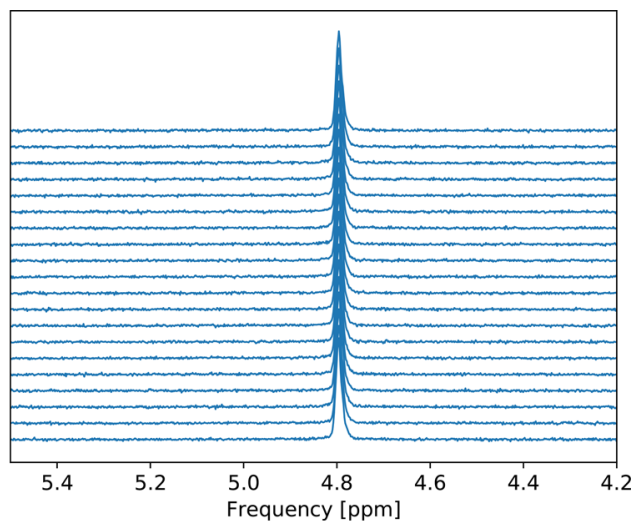

(b) Shim strip

Figure 4. ${ }^{1} \mathrm{H}$ spectra of water shimmed using the regular shims (a) and using the Shim-on-Chip (b). 


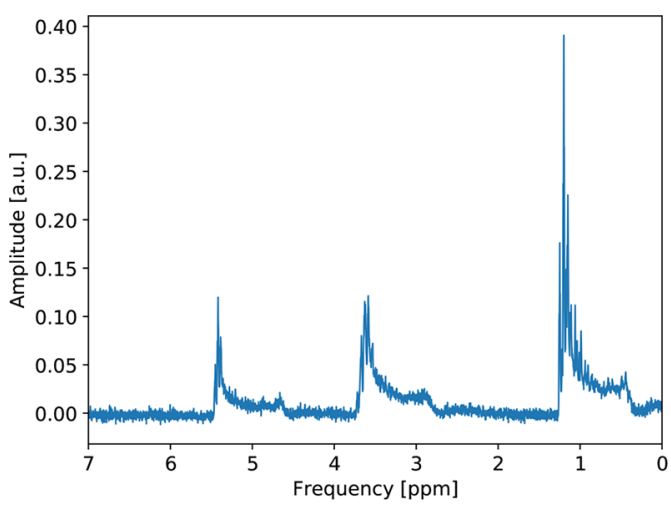

(a) Shim-on-Chip off

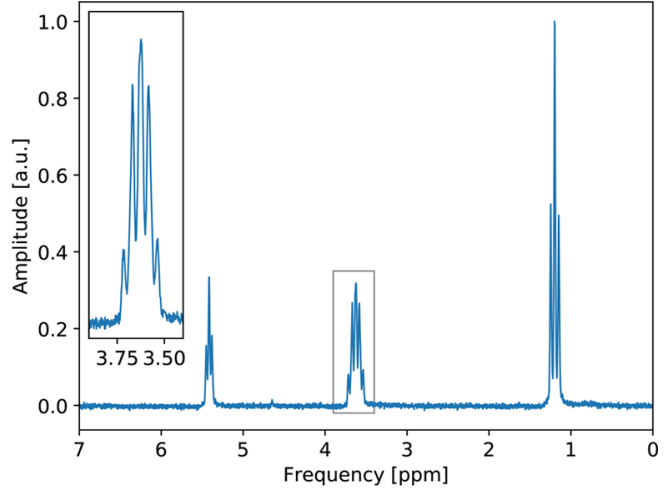

(b) Shim-on-Chip on

Figure 5. ${ }^{1} \mathrm{H}$ spectra of ethanol with the Shim-on-Chip off (a) and on (b).

Shim-on-Chip was optimized on a sample of water using an evolutionary algorithm. This resulted in a line width of $2.2 \mathrm{~Hz}$ at $50 \%$ and $27 \mathrm{~Hz}$ at $0.55 \%$. Then the water in the capillary was replaced by 5-hexen-2-one by flow. After stopping the flow, the spectrum in Figure 6 was recorded without any further

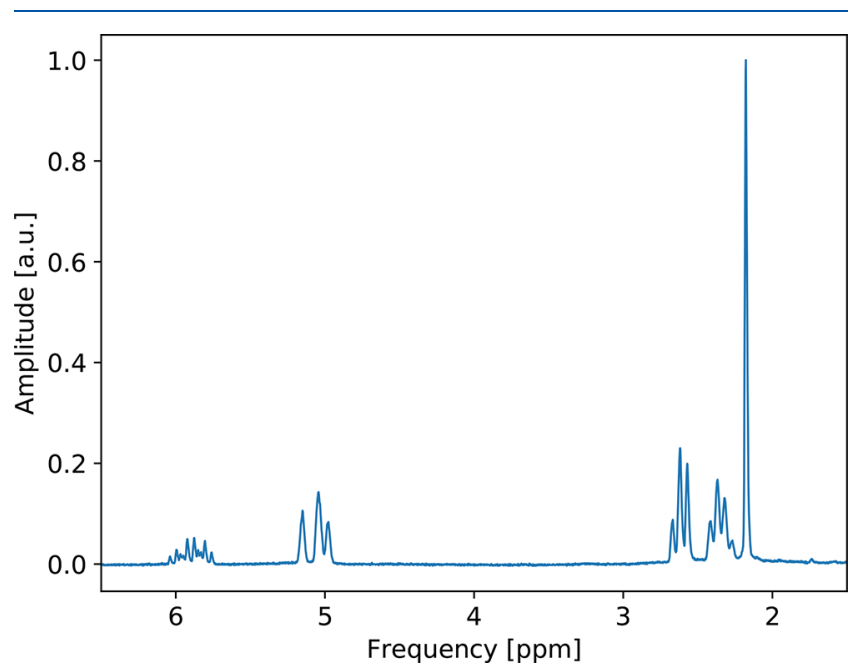

Figure 6. ${ }^{1} \mathrm{H}$ spectrum of 5-hexen-2-one.

shimming. The spectrum of 5-hexen-2-one has the same line width and line shape as the water. This shows that the susceptibility of the sample has a limited effect on the shimming, which is also due to the geometry of the sample in the capillary. Compared to the sensitive region, the sample has the geometry of an infinitely long rod. This means a change in the magnetic susceptibility of the sample will only cause an overall shift in the resonances, but no distortion of the lineshapes.

The Shim-on-Chip does not improve the line width at halfmaximum when compared to conventional shim systems ${ }^{13}$ with results varying for stripline probes fabricated using different procedures. ${ }^{5}$ However, it does show an improvement in the line shape of the resonances. In many applications, microcoil detectors have the issue of a large "foot" at the base of the resonances. This reduces the sensitivity that can be achieved with this type of detector. With the Shim-on-Chip, the lineshapes have a Lorentzian/Gaussian shape.

Additional experiments were performed on a 400 NMR spectrometer detecting off-center samples. The probe used in these experiments is designed for Rapid-Melt DNP NMR experiments. ${ }^{14}$ This means the NMR detector is located offcenter with respect to the main magnet (still within the homogeneous region). The magnet does not contain any regular room temperature shims due to space requirements. In this probe, a ${ }^{1} \mathrm{H}$ spectrum of ${ }^{13} \mathrm{C}$ labeled methanol was acquired in a capillary with an inner diameter of $250 \mu \mathrm{m}$. The stripline and the Shim-on-Chip have similar dimensions as those of the $144 \mathrm{MHz}$ probe.

In Figure 7, the proton spectra are shown for both a shimmed and unshimmed magnet. A decent resolution can be obtained

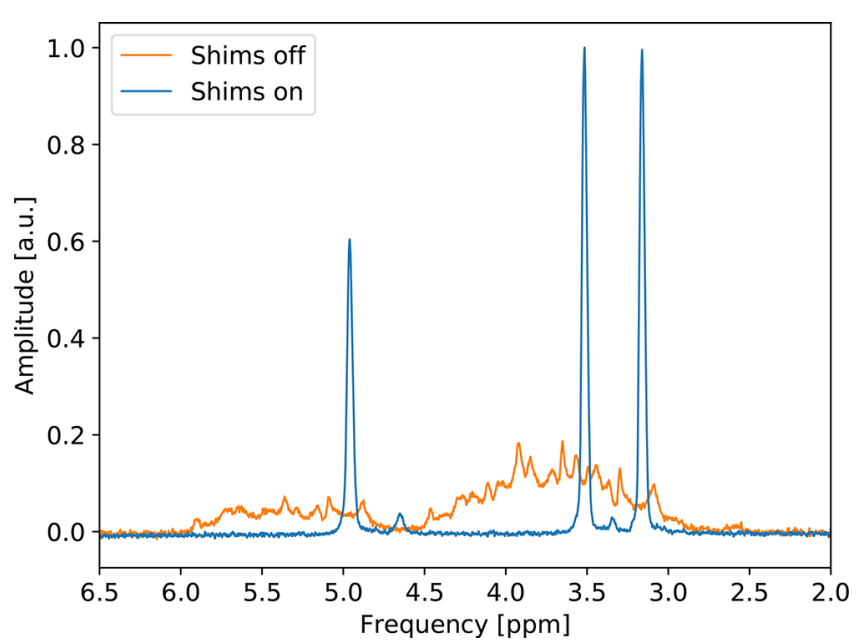

Figure 7. ${ }^{1} \mathrm{H}$ spectrum of carbon labeled methanol with and without shims.

even though the sample is located at the edge of the homogeneous region of the NMR magnet. With a fwhm of 14 $\mathrm{Hz}$, the resolution is not as good as on the $144 \mathrm{MHz}$ spectrometer. This seems to be mainly due to the additional construction parts required for Rapid-Melt experiments. In particular a transfer line for cryogenic liquids made of stainless steel and brass.

The advantage of the Shim-on-Chip is that the shim profiles are always centered on the sample. This means the shims can always create the desired polynomial shim profiles over the detected volume. These profiles have an intuitive effect on the line shape, which simplifies the shimming process on off-center NMR detectors. 


\section{CONCLUSIONS}

A new method of shimming microliter NMR samples has been developed. The Shim-on-Chip is particularly suited for long and thin samples, e.g., contained in capillaries. Shimming a microliter sample using regular shim coils can be troublesome. Because of the small size of the sample, the gradients are generally larger, which requires large currents through the shim coils. In this manuscript we have demonstrated the use of the Shim-on-Chip on a stripline detector, but it can be used in any system where the sample has a near 1D geometry. This is particularly relevant for microfluidic applications where samples are generally long and thin.

Another advantage of the Shim-on-Chip is that it is always centered on the active sample region. This is unlike regular shims where it is often difficult to position the microliter sample at the exact center of the shim coils. Shimming is more complicated with a sample located off-center in the shim coils, because of mixing of the basis functions. ${ }^{6}$

With the Shim-on-Chip system we have reached line widths of just over $2 \mathrm{~Hz}$ at $50 \%$ and $27 \mathrm{~Hz}$ at $0.55 \%$ on a $144 \mathrm{MHz}$ NMR spectrometer. In particular the line shape has been improved with the Shim-on-Chip when compared to previous experiments. ${ }^{13}$ The top and bottom connecting planes of the stripline give rise a large "foot" and makes the line shape asymmetrical. These effects can be compensated using the Shim-on-Chip.

The Shim-on-Chip requires far less space in the magnet compared to regular shims. This is advantageous for complicated setups that require the entire bore space of the magnet, as is the case in the Rapid-Melt DNP experiments. However, the Shim-on-Chip approach may also be useful in reducing the bore size of the magnet. One could think of using this system in combination with a permanent NMR magnet.

It can even be possible to create a pulsed field gradient using a flat ribbon cable. The 16 channel DAC could be replaced by resistors which are inversely proportional to the $n=1$ values in Table S1 in the Supporting Information. All lines could then be driven by a single pulsed dc source. The low inductance of the parallel wires could be useful in decreasing the time needed to switch the field gradient on or off.

\section{ASSOCIATED CONTENT}

\section{S Supporting Information}

The Supporting Information is available free of charge on the ACS Publications website at DOI: 10.1021/acs.analchem.8b02284.

Schematics of the Shim-on-Chip, photographs of the probe head equipped with shim and the shimbox, and currents and field profiles of optimized $z^{n}$ shim profiles (PDF)

\section{AUTHOR INFORMATION}

\section{Corresponding Author}

*E-mail: a.kentgens@nmr.ru.nl.

\section{ORCID}

A. P. M. Kentgens: 0000-0001-5893-4488

Notes

The authors declare no competing financial interest.

\section{ACKNOWLEDGMENTS}

This project is part of the TA-COAST Project 053.21.115 (SFCNMR). The authors would like to acknowledge Gerrit Janssen and Hans Janssen for constructing the probes used in this project.

\section{REFERENCES}

(1) Konzbul, P.; Sveda, K. Meas. Sci. Technol. 1995, 6, 1116-1123.

(2) Golay, M. J. E. Rev. Sci. Instrum. 1958, 29, 313-315.

(3) Anderson, W. A. Rev. Sci. Instrum. 1961, 32, 241-250.

(4) Roméo, F.; Hoult, D. I. Magn. Reson. Med. 1984, 1, 44-65.

(5) Oosthoek-de Vries, A. J.; Bart, J.; Tiggelaar, R. M.; Janssen, J. W. G.; van Bentum, P. J. M.; Gardeniers, H. J. G. E.; Kentgens, A. P. M. Anal. Chem. 2017, 89, 2296-2303.

(6) Hoult, D. J. Magn. Reson. (1969-1992) 1987, 73, 174-177.

(7) van Bentum, P. J. M.; Janssen, J. W. G.; Kentgens, a. P. M.; Bart, J.; Gardeniers, J. G. E. J. Magn. Reson. 2007, 189, 104-13.

(8) Nelder, J. A.; Mead, R. Computer Journal 1965, 7, 308-313.

(9) Ernst, R. R. Rev. Sci. Instrum. 1968, 39, 998-1012.

(10) Storn, R.; Price, K. Journal of Global Optimization 1997, 11, 341359.

(11) Prammer, M. G.; Haselgrove, J. C.; Shinnar, M.; Leigh, J. S. J. Magn. Reson. (1969-1992) 1988, 77, 40-52.

(12) Weiger, M.; Speck, T. Encyclopedia of Magnetic Resonance; John Wiley \& Sons, Ltd.: Chichester, U.K., 2011.

(13) Bart, J.; Janssen, J. W. G.; van Bentum, P. J. M.; Kentgens, a. P. M.; Gardeniers, J. G. E. J. Magn. Reson. 2009, 201, 175-85.

(14) Sharma, M.; Janssen, G.; Leggett, J.; Kentgens, A.; van Bentum, P. J. Magn. Reson. 2015, 258, 40-48. 